\title{
HOMOLOGY COBORDISMS, LINK CONCORDANCES, AND HYPERBOLIC 3-MANIFOLDS
}

BY

\author{
ROBERT MYERS ${ }^{1}$
}

\begin{abstract}
Let $M_{0}^{3}$ and $M_{1}^{3}$ be compact, oriented 3-manifolds. They are homology cobordant (respectively relative homology cobordant) if $\partial M_{i}^{3}=\varnothing$ (resp. $\partial M_{i}^{3} \neq \varnothing$ ) and there is a smooth, compact oriented 4-manifold $W^{4}$ such that $\partial W^{4}=M_{0}^{3}-M_{1}^{3}$ (resp. $\left.\partial W^{4}=M_{0}^{3}-M_{1}^{3}\right) \cup\left(M_{i}^{3} \times[0,1]\right)$ and $H_{*}\left(M_{i}^{3} ; \mathbf{Z}\right) \rightarrow H_{*}\left(W^{4} ; \mathbf{Z}\right)$ are isomorphisms, $i=0,1$.

THEOREM. Every closed, oriented 3-manifold is homology cobordant to a hyperbolic 3-manifold.

THEOREM. Every compact, oriented 3-manifold whose boundary is nonempty and contains no 2-spheres is relative homology cobordant to a hyperbolic 3-manifold.

Two oriented links $L_{0}$ and $L_{1}$ in a 3-manifold $M^{3}$ are concordant if there is a set $A^{2}$ of smooth, disjoint, oriented annuli in $M \times[0,1]$ such that $\partial A^{2}=L_{0}-L_{1}$, where $L_{i} \subseteq M^{3} \times\{i\}, i=0,1$.

THEOREM. Every link in a compact, oriented 3-manifold $M^{3}$ whose boundary contains no 2-spheres is concordant to a link whose exterior is hyperbolic.

COROLlaRY. Every knot in $S^{3}$ is concordant to a knot whose exterior is hyperbolic.
\end{abstract}

1. Introduction. Two closed, oriented 3-manifolds $M_{0}^{3}$ and $M_{1}^{3}$ are homology cobordant if there is a compact, oriented 4-manifold $W^{4}$ such that $\partial W^{4}=M_{0}^{3}-M_{1}^{3}$ and the inclusion induced homomorphisms $H_{*}\left(M_{i}^{3} ; \mathbf{Z}\right) \rightarrow H_{*}\left(W^{4} ; \mathbf{Z}\right)$ are isomorphisms. The set of homology cobordism classes of homology 3-spheres forms an abelian group $\theta_{3}^{H}$, with addition induced by connected sum. At present all that is known of the structure of $\theta_{3}^{H}$ is the existence of an epimorphism $\mu: \theta_{3}^{H} \rightarrow \mathbf{Z}_{2}$, the Rochlin invariant. This group has in recent years become an object of intense study, due to the theorem of Galewski and Stern [2] and Matumoto [9] that all closed topological $n$-manifolds, $n \geqslant 6$, admit simplicial triangulations if and only if there exists $\left\{M^{3}\right\} \in \theta_{3}^{H}$ such that $\mu\left\{M^{3}\right\} \neq 0$ and $2\left\{M^{3}\right\}=0$.

It is therefore of some interest to find a restricted set of "nice" 3-manifolds to represent all the homology cobordism classes. The first result in this direction is due to Livingston [8], who proved that every closed, oriented 3-manifold is homology cobordant to a Haken manifold. In this paper we further restrict the set of representatives by proving that every closed, oriented 3-manifold is homology cobordant to a hyperbolic 3-manifold (Theorem 5.1). In addition the analogous

Received by the editors April 5, 1982.

1980 Mathematics Subject Classification. Primary 57N10; Secondary 57M40, 57M25.

Key words and phrases. 3-manifold, hyperbolic 3-manifold, knot, link, tangle, homology cobordism, knot concordance, link concordance.

'This research was partially supported by NSF Grant MCS-8002524. 
relative result (Theorem 7.1) is proven for compact, oriented 3-manifolds with boundary.

Two oriented links $L_{0}$ and $L_{1}$ in a 3-manifold $M^{3}$ are concordant if they can be joined by disjoint smooth annuli in $M^{3} \times[0,1]$, where $L_{i} \subseteq M \times\{i\}$. The set of concordance classes of knots in $S^{3}$ forms the classical knot concordance group $\bigodot_{1}$, an abelian group with addition induced by composition. Although much more is known about $C_{1}$ than about $\theta_{3}^{H}$ its structure has not yet been completely determined. Thus it may be desirable to find an analogous set of nice representatives. Kirby and Lickorish [7] and Livingston [8] have shown that every knot in $S^{3}$ is concordant to a prime knot. In this paper we show that every link in a compact, oriented 3-manifold whose boundary contains no 2 -spheres is concordant to a link whose exterior is hyperbolic (Theorem 7.2).

We shall always deal with hyperbolic 3-manifolds by means of Thurston's Theorem, which asserts that simple Haken manifolds are hyperbolic. It is thus necessary to develop techniques for constructing simple Haken manifolds and homology cobordisms. These matters, along with definitions and notion, are treated in $\$ 2$. The material in that section as well as others depends heavily on a previous paper [11] in which the author proved that a compact, connected, orientable 3-manifold whose boundary contains no 2-spheres contains a knot with (simple Haken) hyperbolic exterior. Using this result we give in $\$ 3$ a short proof of Livingston's theorem as well as a generalization to manifolds with boundary.

In $\$ 4$ we prove the existence of a disjoint set of properly embedded arcs in a 3-cell (an " $n$-tangle") having a (simple Haken) hyperbolic exterior. This result and the notion of concordant $n$-tangles form the two key ideas in the proof of Theorem 5.1 which is given in $\$ 5$.

In $\S 6$ the main result of [11] is modified to show that every compact, connected, orientable 3-manifold whose boundary contains no 2-spheres contains a properly embedded arc whose exterior is (simple Haken) hyperbolic. This is the principal fact needed to establish the results on relative homology cobordisms (Theorem 7.1) and link concordances (Theorem 7.2) given in $\$ 7$.

The reader should be warned that the proofs of Propositions 4.1 and 6.1, which establish the existence of atoroidal tangles and tunnels and occupy all of $\$ \S 4$ and 6 , are quite long and technical. It may be advisible to skip these proofs on a first reading in order to concentrate on the applications of these results to homology cobordisms and link concordances.

2. Preliminaries. We shall work throughout in either the PL or smooth category; all manifolds are orientable and are assumed compact unless otherwise indicated. Submanifolds are assumed to be locally flat PL or smooth and, unless the contrary is evident, to be properly embedded in or contained in the boundary of their ambient manifolds. The exterior of a subcomplex or submanifold is the closure of the complement of a regular neighborhood.

A knot $K$ in a 3-manifold $M$ is a simple closed curve in the interior of $M$. A link $L$ is a finite disjoint set of knots in $M$. 
The reader is referred to $[4,5,6$ and 14] for the definitions of incompressible and boundary-imcompressible surfaces, compressing and boundary-compressing disks, parallel surfaces, boundary-parallel surfaces, and parallelisms, and of irreducible, boundary-irreducible, and sufficiently large 3-manifolds. The expressions "surface in a 3-manifold" and "surface in the boundary of a 3-manifold" are used as in [14].

A 3-manifold pair $(M, F)$ consists of a 3-manifold $M$ and a surface $F$ in $\partial M$. $(M, F)$ is irreducible if $M$ is irreducible and $F$ is incompressible in $M$. A connected surface $G$ in $M$ with $\partial G$ in $F$ is $F$-compressible if there is a boundary-compressing disk $D$ for $G$ with $\partial D$ in $F \cup G$. $G$ is $F$-parallel if it is parallel to a surface in $F$. The proofs of the following two lemmas are left to the reader.

2.1 Lemma. Let $(M, F)$ be an irreducible 3-manifold pair. Then every F-compressible annulus in $M$ is F-parallel.

2.2 Lemma. Let $\left(M_{0}, F\right)$ be an irreducible 3-manifold pair and $M_{1}$ an irreducible 3-manifold such that $M_{0} \cap M_{1}=\partial M_{0} \cap \partial M_{1}$ is a disjoint set of disks missing $F$. Then $\left(M_{0} \cup M_{1}, F\right)$ is an irreducible 3-manifold pair.

A compact, orientable, irreducible, boundary-irreducible, sufficiently large 3manifold is called a Haken manifold. A compact, orientable, irreducible, boundaryirreducible 3-manifold $M$ is called simple if every incompressible annulus and torus in $M$ is boundary-parallel.

A compact 3-manifold $M$ is hyperbolic if the complement of the torus components of $\partial M$ has a complete Riemannian metric with finite volume and constant negative sectional curvature with respect to which the nontorus components of $\partial M$ are totally geodesic.

We shall always deal with hyperbolic 3-manifolds through the medium of

2.3 The Monster (Thurston's TheOrem). Simple Haken manifolds are hyperbolic.

We shall need some sufficient conditions under which the union of two 3-manifolds along incompressible surfaces in their boundaries is simple and Haken. The following definitions are taken from $\S 3$ of [11], in which the reader can find proofs of the next two lemmas. Let $(M, F)$ be a 3-manifold pair.

$(M, F)$ has Property $A$ if

(1) $(M, F)$ and $(M, \overline{\partial M-F})$ are irreducible 3-manifold pairs,

(2) no component of $F$ is a disk or 2-sphere, and

(3) every disk $D$ in $M$ with $D \cap F$ a single arc is boundary-parallel.

$(M, F)$ has Property $B^{\prime}$ if

(1) $(M, F)$ has Property $A$,

(2) no component of $F$ is an annulus or torus,

(3) every incompressible annulus $A$ in $M$ with $\partial A \cap \partial F=\varnothing$ is boundary-parallel, and

(4) every incompressible torus in $M$ is boundary-parallel.

$(M, F)$ has Property $C^{\prime}$ if

(1) $(M, F)$ has Property $\mathrm{B}^{\prime}$, and 
(2) every disk $D$ in $M$ with $D \cap F$ a pair of disjoint arcs is boundary-parallel.

Now suppose $M=M_{0} \cup M_{1}$, where $M_{0}$ and $M_{1}$ are compact, orientable 3-manifolds and $F=M_{0} \cap M_{1}=\partial M_{0} \cap \partial M_{1}$ is a compact 2-manifold.

2.4 Lemma. If $\left(M_{0}, F\right)$ and $\left(M_{1}, F\right)$ have Property $\mathrm{A}$, then $M$ is Haken. In particular, if $M_{0}$ and $M_{1}$ are Haken, $F,\left(\partial M_{0}-F\right)$ and $\left(\partial M_{1}-F\right)$ are incompressible, and $F$ has no disk or 2-sphere components, then $M$ is Haken.

2.5 Lemma. If $\left(M_{0}, F\right)$ has Property $\mathrm{B}^{\prime}$ and $\left(M_{1}, F\right)$ has Property $\mathrm{C}^{\prime}$, then $M$ is simple and Haken. In particular, if $M_{0}$ and $M_{1}$ are simple Haken manifolds, $F$, $\left(\mathrm{\partial} M_{0}-F\right)$ and $\left(\mathrm{\partial} M_{1}-F\right)$ are incompressible, and no component of $F$ is a disk, 2-sphere, annulus, or torus, then $M$ is a simple Haken manifold.

A homology cobordism is a triple $\left(W^{4} ; M_{0}^{3}, M_{1}^{3}\right)$, where $M_{0}^{3}$ and $M_{1}^{3}$ are closed, oriented 3-manifolds, and $W^{4}$ is a compact, oriented 4-manifold such that $\partial W^{4}=$ $M_{0}^{3}-M_{1}^{3}$ and the inclusion induced homomorphisms $H_{*}\left(M_{i}^{3} ; \mathbf{Z}\right) \rightarrow H_{*}\left(W^{4} ; \mathbf{Z}\right)$, $i=0,1$, are isomorphisms. A relative homology cobordism is a triple $\left(W^{4} ; M_{0}^{3}, M_{1}^{3}\right)$ where $M_{0}^{3}$ and $M_{1}^{3}$ are compact, oriented 3-manifolds with nonempty, homeomorphic boundaries, and $W^{4}$ is a compact, oriented 4-manifold such that $\partial W^{4}=\left(M_{0}^{3}-\right.$ $\left.M_{1}^{3}\right) \cup\left(\partial M_{0}^{3} \times[0,1]\right)$ and the inclusion induced homomorphisms $H_{*}\left(M_{i}^{3} ; \mathbf{Z}\right) \rightarrow$ $H_{*}\left(W^{4} ; \mathbf{Z}\right), i=0,1$, are isomorphisms.

Suppose $\left(P^{4} ; X_{0}^{3}, X_{1}^{3}\right)$ and $\left(Q^{4} ; Y_{0}^{3}, Y_{1}^{3}\right)$ are relative homology cobordisms. Let $F^{2}$ and $G^{2}$ be surfaces in $\partial X_{0}^{3}$ and $\partial Y_{0}^{3}$, respectively. Suppose $\phi: F^{2} \rightarrow G^{2}$ is a homeomorphism. Let $W^{4}=P^{4} \cup_{\phi \times \text { id }} Q^{4}, M_{0}^{3}=X_{0}^{3} \cup_{\phi \times\{0\}} Y_{0}^{3}$, and $M_{1}^{3}=X_{1}^{3}$ $\cup_{\phi \times\{1\}} Y_{1}^{3}$. We denote this construction by

$$
\left(W^{4} ; M_{0}^{3}, M_{1}^{3}\right)=\left(P^{4} ; X_{0}^{3}, X_{1}^{3}\right) \cup_{\phi}\left(Q^{4} ; Y_{0}^{3}, Y_{1}^{3}\right) \text {. }
$$

2.6 Lemma. If $F^{2}=\partial X_{0}^{3}$, then $\left(W^{4} ; M_{0}^{3}, M_{1}^{3}\right)$ is a homology cobordism. If $F^{2} \neq$ $\partial X_{0}^{3}$, then $\left(W^{4} ; M_{0}^{3}, M_{1}^{3}\right)$ is a relative homology cobordism.

Proof. Consider, for $i=0,1$, the commutative diagram

$$
\begin{array}{ccccccc}
\rightarrow & H_{j}\left(X_{i}^{3}\right) \oplus H_{j}\left(Y_{i}^{3}\right) & \rightarrow & H_{j}\left(M_{i}^{3}\right) & \rightarrow & H_{j-1}\left(F^{2} \times\{i\}\right) & \rightarrow \\
& \downarrow & & \downarrow & & \downarrow \\
& \rightarrow \quad H_{j}\left(P^{4}\right) \oplus H_{j}\left(Q^{4}\right) & \rightarrow & H_{j}\left(W^{4}\right) & \rightarrow & H_{j-1}\left(F^{2} \times[0,1]\right) & \rightarrow
\end{array}
$$

where the horizontal rows are Mayer-Vietoris sequences and the vertical homomorphisms are inclusion induced. The first and third of these are clearly isomorphisms. Thus by the Five Lemma so is the second.

Now let $G_{0}$ and $G_{1}$ be homeomorphic 1-complexes in a compact, oriented 3-manifold $M^{3}$ such that $G_{0} \cap \partial M^{3}=G_{1} \cap \partial M^{3}$ consists of vertices. A concordance between $G_{0}$ and $G_{1}$ is a triple $\left(A^{2} ; G_{0}, G_{1}\right)$ where $A^{2}$ is homeomorphic to $G_{0} \times[0,1]$ and is embedded in $M^{3} \times[0,1]$ so that $A^{2} \cap\left(M^{3} \times\{i\}\right)=G_{i}$, for $i=0,1$, and $A^{2} \cap\left(\partial M^{3} \times[0,1]\right)=\left(G_{0} \cap \partial M^{3}\right) \times[0,1]$. (The embedding is required to be locally flat PL or smooth. The meaning of this is clear if $G_{0}$ is a 1-manifold. If $G_{0}$ is not a 1-manifold, one can require that the embedding be a product embedding on a neighborhood of the nonmanifold set.) The exterior of $\left(A^{2} ; G_{0}, G_{1}\right)$ is the triple 
$\left(P^{4} ; X_{0}^{3}, X_{1}^{3}\right)$ consisting of the exteriors of $A^{2}, G_{0}$, and $G_{1}$ in $M^{3} \times[0,1], M^{3} \times\{0\}$, and $M^{3} \times\{1\}$, respectively.

2.7 Lemma. $\left(P^{4} ; X_{0}^{3}, X_{1}^{3}\right)$ is a relative homology cobordism.

Proof. The proof is a Mayer-Vietoris sequence and Five Lemma argument similar to that of Lemma 2.6 .

\section{Homology cobordisms to Haken manifolds.}

3.1 Theorem (Livingston [8]). Every closed, oriented 3-manifold $M_{0}^{3}$ is homology cobordant to a Haken manifold $M_{1}^{3}$.

Proof. We may assume that $M_{0}^{3}$ is connected. $M_{0}^{3}$ contains a knot $J$ whose exterior $X_{0}^{3}$ is a Haken manifold. The basic idea behind this fact is due to Bing [1]. A proof of this assertion in its present form can be found in González-Acuña [3], Myers [10, 11], or Row [13].

Let $K_{1}$ be a nontrivial slice knot in $S^{3}$. Then there is a concordance $\left(A^{2} ; K_{0}, K_{1}\right)$ in $\left(S^{3} \times I ; S^{3} \times\{0\}, S^{3} \times\{1\}\right)$, where $K_{0}$ is the trivial knot. By Lemma 2.7 the exterior $\left(Q^{4} ; Y_{0}^{3}, Y_{1}^{3}\right)$ of this concordance is a relative homology cobordism. $Y_{1}^{3}$ is Haken and $Y_{0}^{3}$ is a solid torus. Let $\phi: \partial X_{0}^{3} \rightarrow \partial Y_{0}^{3}$ be a homeomorphism which extends to a homeomorphism from $\left(M_{0}^{3}-X_{0}^{3}\right)$ to $Y_{0}^{3}$. Let $\left(P^{4} ; X_{0}^{3}, X_{1}^{3}\right)=\left(X_{0}^{3} \times\right.$ $\left.[0,1] ; X_{0}^{3} \times\{0\}, X_{0}^{3} \times\{1\}\right)$ be a product relative homology cobordism. Then by Lemma $2.6\left(W^{4} ; M_{0}^{3}, M_{1}^{3}\right)=\left(P^{4} ; X_{0}^{3}, X_{1}^{3}\right) \cup_{\phi}\left(Q^{4} ; Y_{0}^{3}, Y_{1}^{3}\right)$ is a homology cobordism. By Lemma $2.4 M_{1}^{3} \cup_{\phi} Y_{1}^{3}$ is Haken.

3.2 THEOREM. Every compact, oriented 3-manifold $M_{0}^{3}$ such that $\partial M_{0}^{3} \neq \varnothing$ and contains no 2-spheres is relative homology cobordant to a Haken manifold $M_{1}^{3}$.

Proof. We may assume that $M_{0}^{3}$ is connected. In [11] the author proved that every compact, connected, oriented 3-manifold whose boundary contains no 2-spheres (and may be empty) contains a knot $J$ whose exterior is Haken (in fact simple Haken). The remainder of the proof is similar to that of the previous theorem.

4. Atoroidal $n$-tangles. An $n$-tangle is a set $\left\{\lambda_{1}, \ldots, \lambda_{n}\right\}$ of disjoint, properly embedded arcs in a 3-cell $B$. An $n$-tangle space is the exterior of an $n$-tangle in $B$. An $n$-tangle is atoroidal if its $n$-tangle space is simple.

In [11] the author considered the atoroidal 2-tangle in Figure 1, called the true lover's tangle. The $n$-tangle in Figure 2, illustrated for $n=3$, is a generalization of the true lover's tangle called the true lover's $n$-tangle.

\subsection{Proposition. The true lover's n-tangle is atoroidal.}

Proof. By [11] we may assume that $n \geqslant 3$. The true lover's $n$-tangle space can be expressed as the union of $2 n-1$ cubes with handles, as illustrated in Figure 3 . The proof consists in showing that $\left(\cup_{j=1}^{n} P_{2 j-1}, \cup_{k=1}^{2 n} F_{k}\right)$ has Property $\mathrm{B}^{\prime}$ and $\left(\cup_{j=1}^{n-1} P_{2 j}, \cup_{k=1}^{2 n} F_{k}\right)$ has Property $\mathrm{C}^{\prime}$. It is sufficient to prove that $\left(P_{1}, F_{1}\right)$ has Property $\mathrm{B}^{\prime},\left(P_{2}, F_{1} \cup F_{2}\right)$ has Property $\mathrm{C}^{\prime}$, and $\left(P_{3}, F_{2} \cup F_{3}\right)$ has Property $\mathrm{B}^{\prime}$. The first two of these facts were proved in Lemmas 4.6 and 4.9 of [11] (where $P_{2}$ was called $P$ ). 


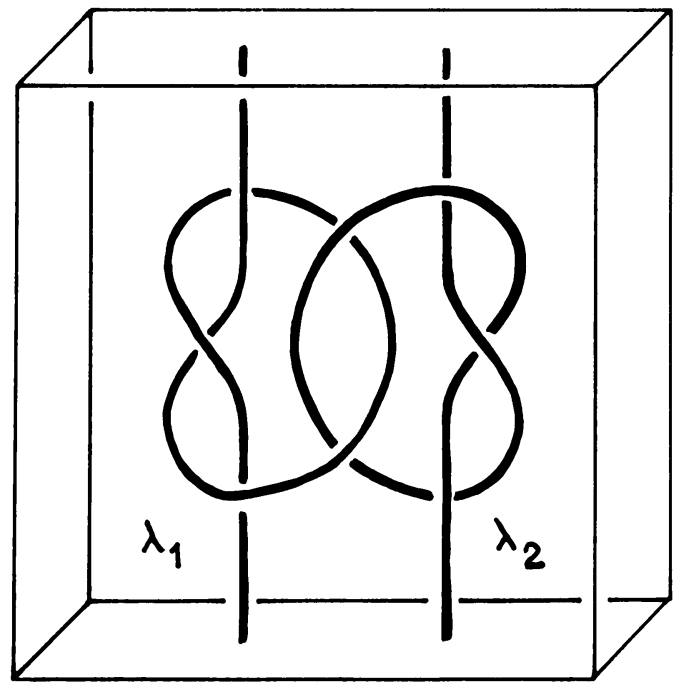

FIGURE 1

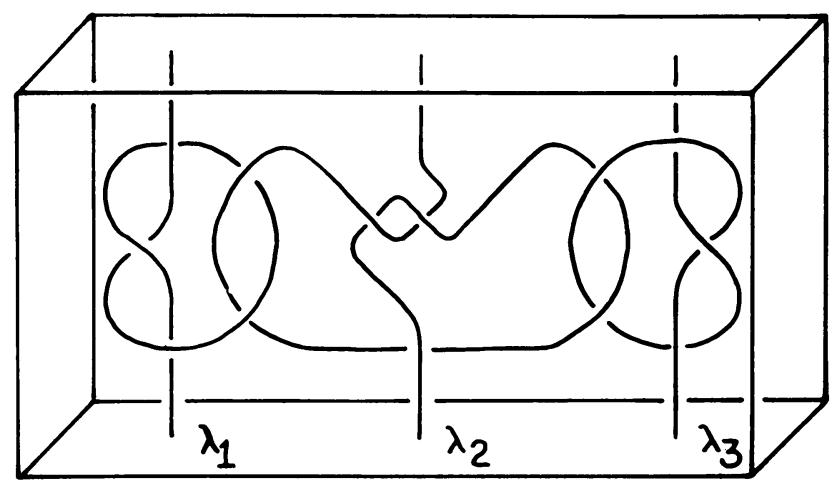

Figure 2

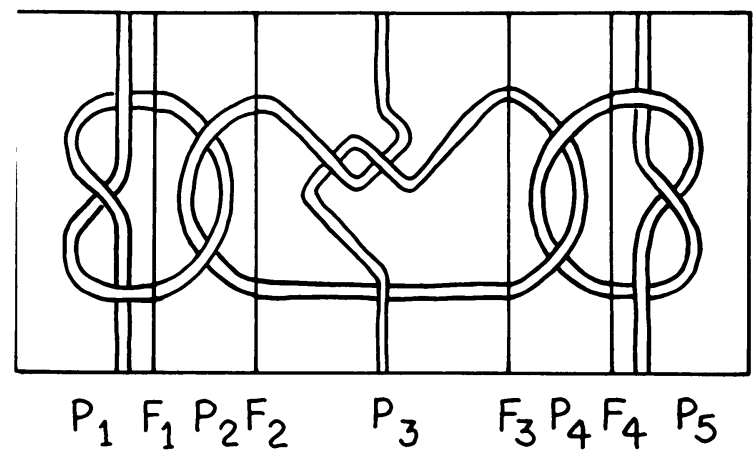

Figure 3 
$\partial P_{3}$ is the union of the planar surfaces $F_{2}, F_{3}$, and $G_{3}$, and the annuli $U_{a}, U_{b}$, and $U_{c}$ shown in Figure 4. $\pi_{1}\left(P_{3}\right)$ is free on $a, b$ and $c, \pi_{1}\left(F_{2}\right)$ is free on $a$ and $b, \pi_{1}\left(F_{3}\right)$ is free on $b^{\prime}$ and $c^{\prime}$, and $\pi_{1}\left(G_{3}\right)$ is free on $a^{\prime}, c$, and $d$. The following relations hold: $a^{\prime}=a c a c^{-1} a^{-1}, d=a b, b^{\prime}=c^{-1} b c, c^{\prime}=a c a^{-1}$, and $d^{\prime}=a c a^{-1} c^{-1} b c$.

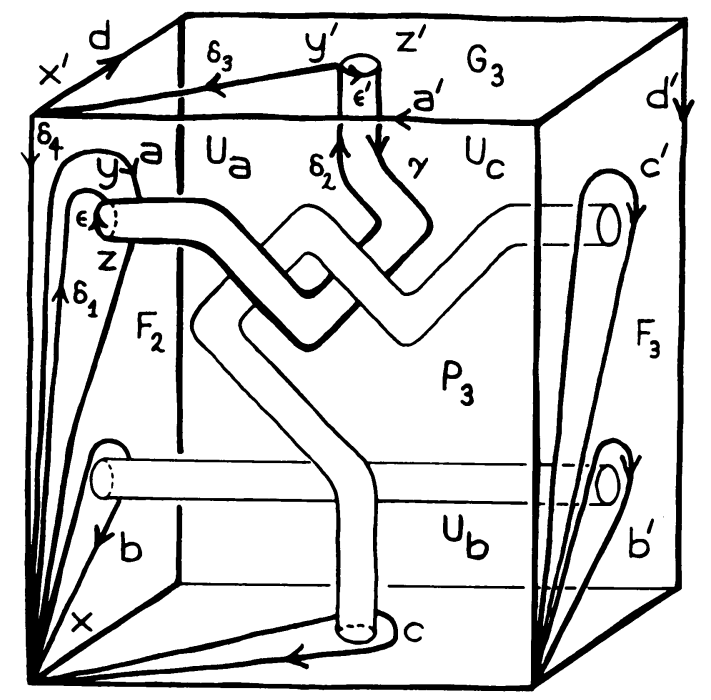

Figure 4

In the following algebraic lemmas $W^{\prime}$ denotes a word in $a^{\prime}, c$, and $d$, and $W$ a word in $a, b$, and $c . V \equiv W$ means that the words $V$ and $W$ are identical, while $V=W$ means that they determine the same element of $\pi_{1}\left(P_{3}\right)$. Every nontrivial reduced word $W^{\prime}$ determines a nontrivial reduced word $W$, which is called a $G$-word. Thus $G_{3}$ is incompressible in $P_{3}$. Let $p$ and $r$ be elements of $\left\{a, b^{-1}\right\}$. Words of the form $p c a^{m} c^{-1} r^{-1}$ or $p r^{-1}$ are called special words. Every $G$-word is a positive power product of $c, c^{-1}$, and special word. (Note that although every such product is an element of $\pi_{1}\left(G_{3}\right)$, not every such product is a $G$-word, since certain juxtapositions result in nonreduced words.)

Let $S, P, Q, R$ be, respectively, a special word, an initial segment of a special word, a $G$-word, and a terminal segment of a special word. $S$ is never a (proper) segment of a special word. One never has $P \equiv R$. Since $S$ does not begin or end in $c^{k}$, this implies that $P$ is never a terminal segment of $Q$, and $R$ is never an initial segment of $Q$. Moreover, if $P \neq a$, then $P$ is never a noninitial segment of $S$, and if $R \nexists a^{-1}, R$ is never a nonterminal segment of $S$. It follows that:

(1) If $X Q Z$ is a $G$-word, then $X$ and $Z$ are $G$-words.

(2) If $X P Z$ is a $G$-word, and $P Z a$, then $X$ and $P Z$ are $G$-words.

(3) If $X R Z$ is a $G$-word, and $R Z a^{-1}$, then $X R$ and $Z$ are $G$-words.

From these facts it follows that the above factorization of a $G$-word is unique.

4.2 Lemma. $\pi_{1}\left(F_{2}\right) \cap \pi_{1}\left(G_{3}\right)=g p(d)$.

Proof. Clearly $g p(d) \subseteq\left(\pi_{1}\left(F_{2}\right) \cap \pi_{1}\left(G_{3}\right)\right)$. Suppose $w \in\left(\pi_{1}\left(F_{2}\right) \cap \pi_{1}\left(G_{3}\right)\right)$ is represented by the $G$-word $W$. Since $w \in \pi_{1}\left(F_{2}\right), W$ does not involve $c$, hence $W$ does not involve $c^{ \pm 1}$ or $p c a^{m} c^{-1} r^{-1}$ and is therefore a power of $d$. 
4.3 LEMMA. $\pi_{1}\left(F_{2}\right) \cap \pi_{1}\left(G_{3}\right) a c a^{-1}=\varnothing$.

Proof. If $v \in \pi_{1}\left(F_{2}\right) \cap \pi_{1}\left(G_{3}\right) a c a^{-1}$, then $v=$ waca $^{-1}$ where $w \in \pi_{1}\left(G_{3}\right)$ and is represented by the $G$-word $W$. Since $v \in \pi_{1}\left(F_{2}\right), W a c a^{-1}$ is not reduced, hence $W$ terminates in $p c a^{m} c^{-1} a^{-1}$ or $b^{-1} a^{-1}$. Therefore the reduction of $W c a^{-1}$ is $W_{0} p c a^{m-1}$ or $W_{0} b^{-1} c^{-1} a^{-1}$, which cannot be in $\pi_{1}\left(F_{2}\right)$, a contradiction.

4.4 LEMMA. $\pi_{1}\left(F_{2}\right) \cap a c^{-1} a^{-1} \pi_{1}\left(G_{3}\right) a c a^{-1}=g p(a)$.

Proof. $g p(a) \subseteq\left(\pi_{1}\left(F_{2}\right) \cap a c^{-1} a^{-1} \pi_{1}\left(G_{3}\right) a c a^{-1}\right)$ because

$$
a^{m}=\left(a c^{-1} a^{-1}\right)\left(a c a^{m} c^{-1} a^{-1}\right)\left(a c a^{-1}\right) .
$$

Suppose $v \in\left(\pi_{1}\left(F_{2}\right) \cap a c^{-1} a^{-1} \pi_{1}\left(G_{3}\right) a c a^{-1}\right)$. Then $v=a c^{-1} a^{-1} w_{a c a^{-1}}$, where $w \in$ $\pi_{1}\left(G_{3}\right)$ is represented by the $G$-word $W$. Let $V$ represent $v$. Then $a c a^{-1} V a c^{-1} a^{-1}=W$. $W$ is the reduction of $a c a^{-1} V a c^{-1} a^{-1}$. If $V \neq a^{m}$, then, since $V$ does not involve $c$, $W \equiv a c V_{0} c^{-1} a^{-1}$, where $V_{0}$ is the reduction of $a^{-1} V a$. $W$ begins and ends in words of the form $p c a^{m} c^{-1} r^{-1}$, which forces $V_{0}$ and hence $V$ to involve $c$, a contradiction.

4.5 LeMmA. If $v \in \pi_{1}\left(P_{3}\right)$ and $v^{n} \in \pi_{1}\left(G_{3}\right), n \neq 0$, then $v \in \pi_{1}\left(G_{3}\right)$.

Proof. We may assume that $v \neq 1$ and $n>1$. Let $V$ and $W$ be reduced words representing, respectively, $v$ and $v^{n}$. Let $S, P, Q, R$ be as above.

Suppose $V^{n}$ is reduced. If $V$ is a $G$-word, then $v \in \pi_{1}\left(G_{3}\right)$. If $V$ is not a $G$-word, then $V \equiv P$ or $Q P$, and so $V^{n} \equiv P^{n}$ or $(Q P)^{n}$, both of which are impossible.

Suppose $V^{n}$ is not reduced. Then $V \equiv X Y \equiv Z X^{-1}$. If $l(X) \geqslant \frac{1}{2} l(V)$, then $V \equiv$ $Z M Y$, where $X \equiv Z M$ and $X^{-1} \equiv M Y$. Since $X^{-1} \equiv M^{-1} Z^{-1}$ and $l(M)=l(M)^{-1}$, $M \equiv M^{-1}$, so that $M^{2}=1$. This implies that $M=1$ because $\pi_{1}\left(P_{3}\right)$ is free. Therefore $V \equiv Z Y \equiv X X^{-1} \equiv 1$, a contradiction. Hence $l(X)<\frac{1}{2} l(V)$ and so $V \equiv X Y X^{-1}$ and $W \equiv X Y^{n} X^{-1}$, which is reduced. Regard $Y^{n}$ as $Y_{1} Y_{2} \cdots Y_{n}$, where each $Y_{i} \equiv Y$. In the hypotheses of the following cases $P$ and $R$ refer, respectively, to initial and terminal segments of special words embedded in $W$ and overlapping with $Y_{1}$ as indicated.

Case 1. $Y_{1} \equiv Q$. Then $Y^{n}$ is a $G$-word, and so $X$ and $X^{-1}$ are $G$-words. Therefore $V \equiv X Q X^{-1}$ is a $G$-word.

Case 2. $Y_{1} \equiv P$. Then $X$ is a $G$-word. Hence $X^{-1}$ and $Y^{n}$ are $G$-words. By the argument for $V^{n}$ reduced, $Y$ is a $G$-word, contradicting $Y_{1} \equiv P$.

Case 3. $Y_{1} \equiv R$. Then $X Y_{1}$ is a $G$-word, and so $Y_{2} \cdots Y_{n} X^{-1}$ is a $G$-word, which is impossible since $Y_{2} \equiv R$.

Case 4. $Y_{1}$ is a noninitial, nonterminal segment of $S$. Then $S \equiv p c a^{m} c^{-1} r^{-1}$. $Y_{2} \equiv Y_{1}$ implies that $Y_{1} \equiv a^{k}$. $W$ reduced implies that $X \equiv X_{0} p c$, where $X_{0}$ is a $G$-word. Hence $V \equiv X_{0} p c a^{k} c^{-1} p^{-1} X_{0}^{-1}$, which is a $G$-word.

Case 5. $Y_{1} \equiv R Q$. Then $X R Q$ is a $G$-word. So $(R Q)^{n-1} X^{-1}$ is a $G$-word, which is impossible.

Case 6. $Y_{1} \equiv Q P$. Then $X$ is a $G$-word. Hence $X^{-1}$ is a $G$-word, and so $P(Q P)^{n-1}$ is a $G$-word, which is impossible.

Case 7. $Y_{1} \equiv R Q P$. Then $W \equiv X R(Q R P)^{n-1} Q P X^{-1}$ implies that $X R, R P$ and $P X^{-1}$ are $G$-words. Thus $V \equiv X R Q P X^{-1}$ is a $G$-word. 
Case 8. $Y_{1} \equiv R P$. Then $W \equiv X(R P)^{n} X^{-1} . X R$ is a $G$-word. If $P Z a$, then $P X^{-1}$ is a $G$-word. If $P \equiv a$, then $R \neq a^{-1}$. So $X(R P)^{n-1} R$ and $P X^{-1}$ are $G$-words. Thus $V \equiv X R P X^{-1}$ is a $G$-word.

This completes the proof.

In the following lemmas, $J_{a}, J_{a^{\prime}}$, etc. denote the component of $\partial F_{2}, \partial G_{3}$, etc. homologous to $a, a^{\prime}$, etc. $f$ and $g$ denote elements of $\pi_{1}\left(F_{2}\right)$ and $\pi_{1}\left(G_{3}\right)$, respectively. Let $F=F_{2} \cup F_{3}$.

\subsection{Lemma. $\left(P_{3}, F\right)$ has Property A.}

ProOF. $P_{3}$ is a cube with handles and is therefore irreducible. No component of $F$ is a disk or 2-sphere. $F$ is incompressible for homological reasons. Thus $U_{a}, U_{b}$, and $U_{c}$ are incompressible. As noted above, $G_{3}$ is incompressible.

Let $\gamma, \delta_{1}, \delta_{2}, \delta_{3}, \delta_{4}, \varepsilon$ and $\varepsilon^{\prime}$ be the arcs and $x, x^{\prime}, y, y^{\prime}$ and $z$ be the points in Figure 4. Let $\delta=\delta_{1} \delta_{2} \delta_{3} \delta_{4}$. Then $[\delta]=a c^{-1} a^{-1}$, and $\varepsilon^{\prime} \gamma \varepsilon^{-1}$ is homotopic to $\delta_{2}^{-1} \operatorname{rel}\left\{y, y^{\prime}\right\}$.

Let $D$ be a disk in $P_{3}$ with $\partial D \cap F$ a single arc $\alpha$. Let $\beta=\overline{\partial D-\alpha}$. Note that there is a homeomorphism of $P_{3}$ which interchanges $F_{2}$ and $F_{3}$, interchanges $U_{a}$ and $U_{c}$, and leaves $U_{b}$ invariant. We may thus assume that $\alpha \subseteq F_{2}$.

Case 1. $\alpha \cap J_{b} \neq \varnothing$.. Then $\beta$ is a $J_{b}$-parallel arc in $U_{b}$. Hence $D$ can be isotoped so that $\partial D \subseteq F_{2}$. By the incompressibility of $F_{2}$ and irreducibility of $P_{3}, D$ is boundaryparallel.

Case 2. $\partial \alpha \subseteq J_{d}$. Isotop $D$ and orient $\partial D$ so that $\alpha$ runs from $x$ to $x^{\prime}$. Then $[\partial D]=\left[\alpha \delta_{4}\right]\left[\delta_{4}^{-1} \beta\right]=f g=1$. Hence $f \in \pi_{1}\left(F_{2}\right) \cap \pi_{1}\left(G_{3}\right)$. By Lemma 4.2, $f=d^{k}$. Since $\alpha \delta_{4}$ is a simple closed curve, $|k| \leqslant 1$. It follows that $\alpha$ is parallel to $\delta_{4}$ or to $\overline{J_{d}-\delta_{4}}$ in $F_{2}$. Thus $D$ can be isotoped so that $\partial D \subseteq G_{3}$. Hence $D$ is boundary-parallel.

Case 3. $\alpha$ runs from $J_{d}$ to $J_{a}$. Isotop $D$ and orient $\partial D$ so that $\alpha$ runs from $x$ to $y$ and $\beta \cap U_{a}=\delta_{2}$. Let $\beta^{\prime}=\beta \cap G_{3}$. Then

$$
[\partial D]=\left[\alpha \delta_{1}^{-1}\right]\left[\delta_{1} \delta_{2} \delta_{3} \delta_{4}\right]\left[\delta_{4}^{-1} \delta_{3}^{-1} \beta^{\prime}\right]=\mathrm{fac}^{-1} a^{-1} g=1 .
$$

So $f=g^{-1} a c a^{-1} \in \pi_{1}\left(F_{2}\right) \cap \pi_{1}\left(G_{3}\right) a c a^{-1}$, contradicting Lemma 4.3. Therefore this case cannot occur.

Case 4. $\partial \alpha \subseteq J_{a}$. Isotop $D$ and orient $\partial D$ so that $\alpha$ runs from $z$ to $y$. We may assume that $\beta=\beta_{1} \beta_{2} \beta_{3}$, where $\beta_{1}=\delta_{2}, \beta_{2}$ runs from $y^{\prime}$ to $z^{\prime}$ in $G_{3}$, and $\beta_{3}=\gamma$. Referring $[\partial D]$ to $x$ via $\delta_{1}$, we have

$$
\begin{aligned}
1 & =[\partial D]=\left[\delta_{1} \beta_{1} \beta_{1} \beta_{3} \alpha \delta_{1}^{-1}\right] \\
& =\left[\delta_{1} \delta_{2} \delta_{3} \delta_{4}\right]\left[\delta_{4}^{-1} \delta_{3}^{-1} \beta_{2}\left(\varepsilon^{\prime}\right)^{-1} \delta_{3} \delta_{4}\right]\left[\delta_{4}^{-1} \delta_{3}^{-1}\left(\varepsilon^{\prime} \gamma \varepsilon^{-1}\right) \delta_{1}^{-1}\right]\left[\delta_{1} \varepsilon \alpha \delta_{1}^{-1}\right] \\
& =[\delta] g[\delta]^{-1} f . \text { So } f^{-1}=a c^{-1} a^{-1} g a c a^{-1} .
\end{aligned}
$$

By Lemma $4.4 f=a^{m}$. Since $\varepsilon \alpha$ is a simple closed curve, $|m| \leqslant 1$ and hence $\alpha$ is parallel to $\varepsilon$ or $\overline{J_{a}-\varepsilon}$ in $F_{2}$. Thus $D$ can be isotoped so that $\partial D \subseteq G_{3}$ and so is boundary-parallel.

4.7 Lemma. $\left(P_{3}, F\right)$ has Property B'. 
Proof. Clearly $P_{3}$ contains no incompressible tori. Suppose $A$ is an incompressible annulus in $P_{3}$ with $\partial A \cap \partial F=\varnothing$. Let $\alpha$ and $\beta$ be the components of $\partial A$. We may assume that $\partial A \subseteq\left(F \cup G_{3}\right)$.

Case 1. $\alpha$ and $\beta$ are parallel in $\partial P_{3}$. Then $\alpha$ and $\beta$ cobound an annulus $A^{\prime}$ in $\partial P_{3} . A$ can be isotoped so that $\alpha$ and $\beta$ lie in the same component of $\left(P_{3}-\partial F\right)$, which we may assume to be $F_{2}$ or $G_{3}$. Let $T^{\prime}$ be the result of isotoping the torus $T=A \cup A^{\prime}$ slightly into Int $P_{3}$. $T^{\prime}$ is compressible in $P_{3}$ and thus bounds either a solid torus $V^{\prime}$ or knot exterior $Q^{\prime}$ in $P_{3}$. If $T^{\prime}$ bounds $Q^{\prime}$, then the boundary of a compressing disk for $T^{\prime}$ must be a meridian of $Q^{\prime}$. Since $A^{\prime}$ lies on the boundary of an obvious 3-cell containing $P_{3}, \alpha$ is homotopic in $P_{3}$ to a meridian of $Q^{\prime}$. This contradicts the incompressibility of $A$ in $P_{3}$. Therefore $T^{\prime}$ bounds $V^{\prime}$, and hence $T$ bounds a solid torus $V$. If $A$ is not parallel to $A^{\prime}$ across $V$, then the core of $V$, referred to the basepoint, represents a root of an element of $\pi_{1}\left(F_{2}\right)$ which does not lie in $\pi_{1}\left(G_{3}\right)$. The first possibility is ruled out by $\pi_{1}\left(F_{2}\right)$ being a free factor of $\pi_{1}\left(P_{3}\right)$. The second is ruled out by Lemma 4.5. Thus $A$ is boundary-parallel.

Case 2. $\alpha$ and $\beta$ are not parallel in $\partial P_{3}$. We show that this cannot happen. For homological reasons, we may assume that $\alpha$ is parallel to $J_{b}$ in $F_{2}$ and $\beta$ separates $J_{a^{\prime}} \cup J_{d}$ from $J_{c} \cup J_{d^{\prime}}$ in $G_{3}$. Regard the disk $E$ in Figure 5 as [0,2] $\times[0,1]$, where $\{0\} \times[0,1] \subseteq U_{a},\{2\} \times[0,1] \subseteq U_{c},[0,1] \times\{0\} \subseteq F_{2},[1,2] \times\{0\} \subseteq G_{3},[0,1] \times$ $\{1\} \subseteq G_{3}$, and $[1,2] \times\{1\} \subseteq F_{2}$. Let $\theta$ be the arc on $\partial P_{3}$ running from $x=\{1\} \times\{0\}$ to $w=\{1\} \times\{1\}$ and $\omega$ the $\operatorname{arc}\{1\} \times[0,1]$ running from $w$ to $x$ shown in Figure 5 . $[\theta \omega]=c a$, which is clearly not in $\pi_{1}\left(G_{3}\right)$.

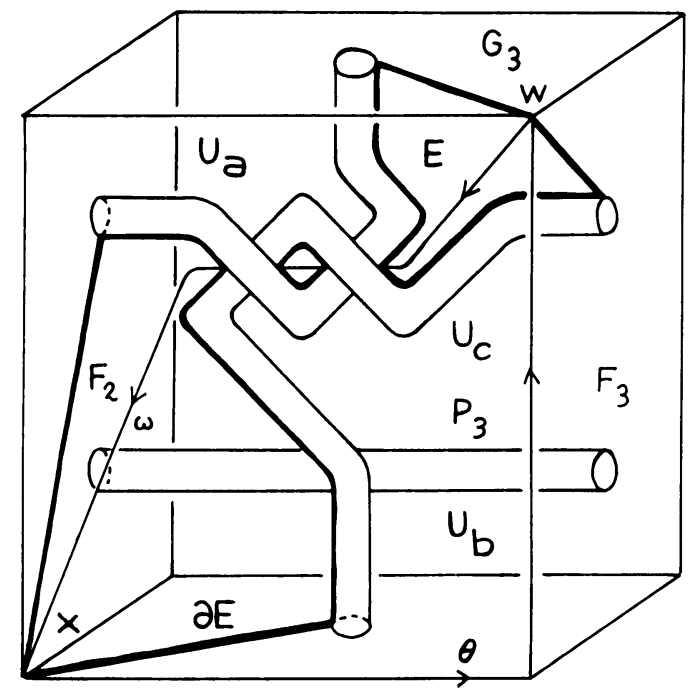

Figure 5

Isotop $A$, keeping $\alpha$ in $F_{2}$ and $\beta$ in $G_{3}$, so that $A$ and $E$ are in general position and meet in a minimal number of components. Since $[0,1] \times\{1\}$ joins $J_{a^{\prime}}$ to $J_{d^{\prime}}$, $A \cap E \neq \varnothing$. 
If each component of $A \cap E$ is parallel in $E$ to an arc in $G_{3}$, then there is a component $\rho$ which cobounds a disk $D_{0}$ on $A$ with an $\operatorname{arc} \rho_{0}$ in $\beta$ and a disk $D_{1}$ on $E$ with an $\operatorname{arc} \rho_{1}$ in $G_{3}$ such that $E \cap$ Int $D_{0}=\varnothing$. By the incompressibility of $G_{3}$ and irreducibility of $P_{3}$, the disk $D_{0} \cup D_{1}$ is $G_{3}$-parallel. It follows that $A$ can be isotoped, keeping $\alpha$ in $F_{2}$ and $\beta$ in $G_{3}$, so as to remove at least $\rho$ from $A \cap E$, contradicting minimality.

Therefore some component $\rho$ of $A \cap E$ runs from [0,1] $\times\{1\}$ to [1,2] $\times\{0\}$. Let $\xi$ be an arc in $[0,1] \times\{1\}$ running from $w$ to the origin of $\rho$, and let $\eta$ be an arc in $[1,2] \times\{0\}$ running from the terminus of $\rho$ to $x$. $\xi \rho \eta$ is homotopic in $E$ to $\omega$, and $\rho$ is homotopic in $A$ to an $\operatorname{arc} \rho^{\prime}$ in $\beta$, both homotopies fixing $x$ and $w$. Therefore $c a=[\theta \omega]=[\theta \xi \rho \eta]=\left[\theta \xi \rho^{\prime} \eta\right] \in \pi_{1}\left(G_{3}\right)$, a contradiction. This completes the proof.

\section{Homology cobordisms to hyperbolic 3-manifolds.}

5.1 THEOREM. Every closed, oriented 3-manifold $M_{0}^{3}$ is homology cobordant to a hyperbolic 3-manifold $M_{1}^{3}$.

The proof depends on

5.2 LEMMA. Every cube with handles $X_{0}^{3}$ (of genus at least one) is relative homology cobordant to a simple Haken (and thus hyperbolic) 3-manifold $X_{1}^{3}$.

Proof. If $X_{0}^{3}$ is a solid torus, then let $X_{1}^{3}$ be the exterior of any nontrivial, simple slice knot in $S^{3}$, e.g. the stevedore's knot [12]. The exterior of a concordance between this knot and the trivial knot is the required homology cobordism.

We therefore assume that $X_{0}^{3}$ has genus $n \geqslant 2$. Let $\left\{\mu_{1}, \ldots, \mu_{n}\right\}$ be the $n$-tangle in the 3-cell $B^{3}$ in Figure 6 with exterior $X_{1}^{3} \cdot\left\{\mu_{1}, \ldots, \mu_{n}\right\}$ is the composition of the true lover's $n$-tangle $\left\{\lambda_{1}, \ldots, \lambda_{n}\right\}$ with its reflection in a plane. As illustrated in Figure 7 , $\left\{\mu_{1}, \ldots, \mu_{n}\right\}$ is concordant to a trivial tangle $\left\{\mu_{1}^{\prime}, \ldots, \mu_{n}^{\prime}\right\}$ whose exterior can be identified with $X_{0}^{3}$. By Lemma 2.7 the exterior $\left(P^{4} ; X_{0}^{3}, X_{1}^{3}\right)$ of this concordance is a relative homology cobordism.

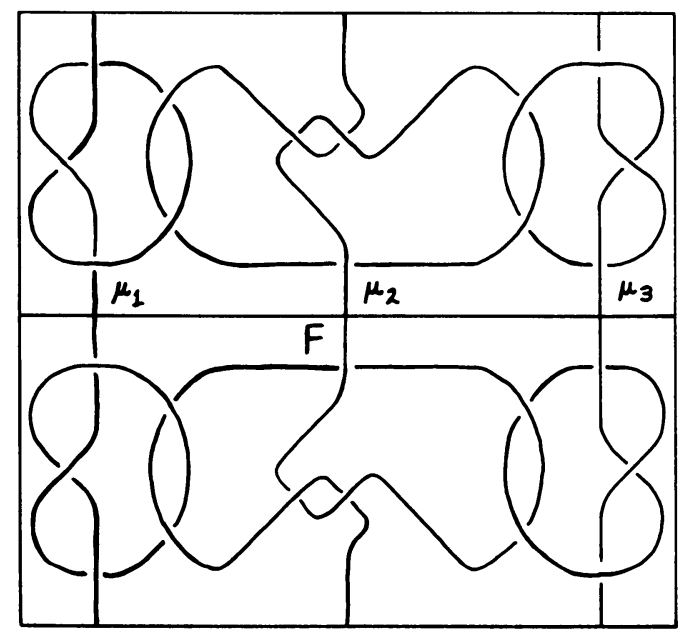

FIGURE 6 
$X_{1}^{3}=Q^{3} \cup\left(-Q^{3}\right)$, where $Q^{3}$ is the true lover's $n$-tangle space and $Q^{3} \cap\left(-Q^{3}\right)=F$ is the planar surface in Figure 6. By Proposition $4.1 Q^{3}$ is simple. Since $F$ is clearly incompressible in $Q^{3}$ and $-Q^{3},\left(Q^{3}, F\right)$ and $\left(-Q^{3}, F\right)$ have Property $C^{\prime}$. Since $Q^{3}$ is clearly Haken, Lemma 2.5 implies that $X_{1}^{3}$ is simple and Haken.

(a)

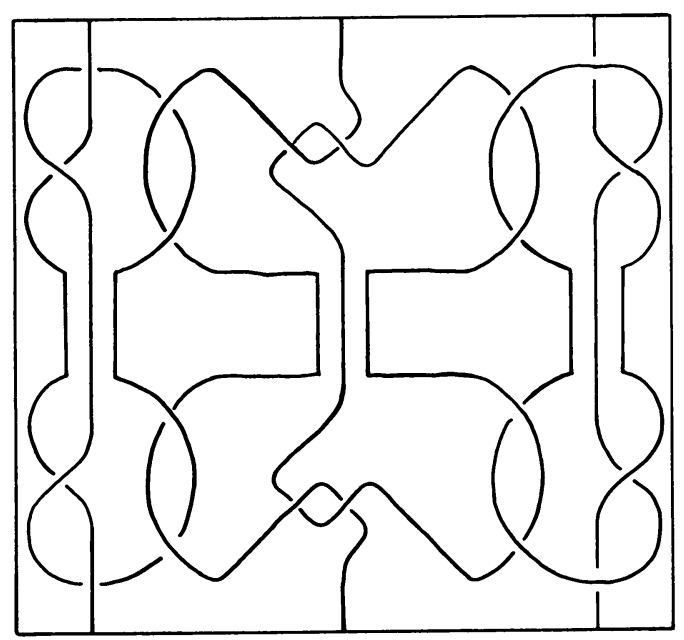

(b)

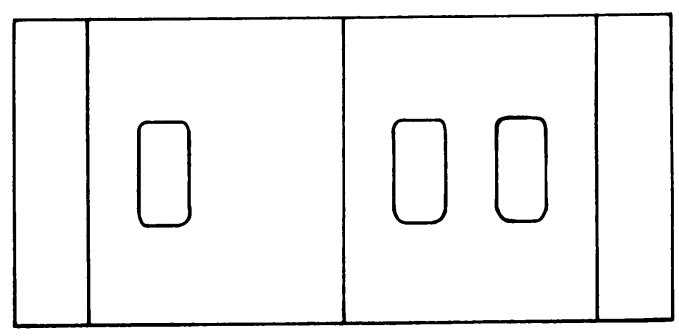

(c)

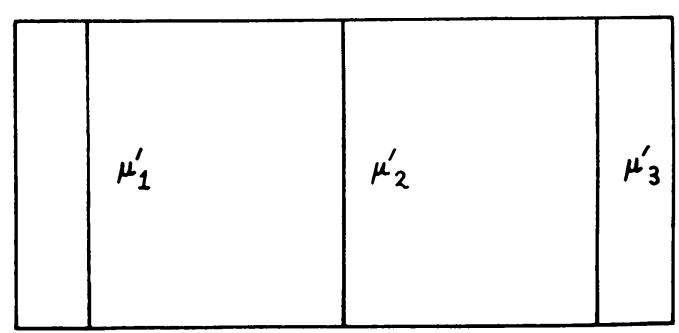

FIGURE 7

Proof of Theorem 5.1. We may assume that $M_{0}^{3}$ is connected. Let $X_{0}^{3} \cup_{\phi} Y_{0}^{3}$ be a Heegaard splitting of $M_{0}^{3}$, i.e. $X_{0}^{3}$ and $Y_{0}^{3}$ are cubes with handles, and $\phi: \partial X_{0}^{3} \rightarrow \partial Y_{0}^{3}$ is a homeomorphism under whose identification one obtains $M_{0}^{3}$. We may assume, by adding trivial handles if necessary, that the splitting has genus at least two. Let $\left(P^{4} ; X_{0}^{3}, X_{1}^{3}\right)$ and $\left(Q^{4} ; Y_{0}^{3}, Y_{1}^{3}\right)$ be relative homology cobordisms, where $X_{1}^{3}$ and $Y_{1}^{3}$ are simple Haken manifolds. Let $\left(W^{4} ; M_{0}^{3}, M_{1}^{3}\right)=\left(P^{4} ; X_{0}^{3}, X_{1}^{3}\right) \cup_{\phi}\left(Q^{4} ; Y_{0}^{3}, Y_{1}^{3}\right)$. By Lemma $2.6\left(W^{4} ; M_{0}^{3}, M_{1}^{3}\right)$ is a homology cobordism. By Lemma $2.5 M_{1}^{3}$ is simple and Haken and therefore hyperbolic. 


\section{Atoroidal tunnels.}

6.1 Proposition. Let $M$ be a compact, connected, orientable 3-manifold such that $\partial M \neq \varnothing$ and contains no 2-spheres. Let $F$ be a component of $\partial M$. Then $M$ contains $a$ properly embedded arc $\tilde{J}$ such that $\partial \tilde{J} \subseteq F$ and the exterior of $\tilde{J}$ is a simple Haken manifold.

The proof will be a modification of $\$ \S 5$ and 6 of [11], in which the author constructed a knot in $M$ whose exterior is a simple Haken manifold. Let $C=\partial M \times$ $[0,1]$ be a collar on $\partial M$, with $\partial M=\partial M \times\{1\}$. Let $N=\overline{(} M-C)$. In Lemma 5.1 of [11] it was shown that $N$ admits a special handle decomposition $\left\{h_{i}^{k}\right\}$, i.e. a handle decomposition [14] such that:

(1) $h_{i}^{k} \cap \partial N$ and $h_{i}^{k} \cap h_{j}^{\prime}, i \neq j$, are each either empty or connected,

(2) each 0 -handle $h_{i}^{0}$ meets exactly four 1-handles and six 2-handles,

(3) each 1-handle $h_{j}^{1}$ meets exactly two 0 -handles and three 2-handles,

(4) each pair $h_{k}^{2}, h_{l}^{2}$ of 2-handles either

(a) meets no common 0 - or 1-handle, or

(b) meets exactly one common 0 -handle and no common 1-handle, or

(c) meets exactly one common 1-handle and two common 0 -handles,

(5) the complement of any 0 -handle in the union $H^{\prime}$ of the 0 - and 1-handles is connected, and

(6) the union of any 0 -handle with the union $H^{\prime \prime}$ of the 2- and 3-handles is a cube with handles which meets $\partial N$ in a disjoint collection of disks.

Let $C_{i}^{k}$ be the core of the handle $h_{i}^{k}$. Let $Z=H^{\prime \prime} \cup C, R_{i}=h_{i}^{0} \cap Z$, and $S_{j}=h_{j}^{1} \cap Z$.

In [11] it was shown that $\cup C_{j}^{1}$, together with atoroidal 2-tangles in the $h_{i}^{0}$, formed a knot $J$ whose exterior is a simple Haken manifold. The arc $\tilde{J}$ will be constructed by deleting one $C_{0}^{1}$ whose 1-handle $h_{0}^{1}$ meets $F \times\{0\}$ and connecting the endpoints to $F$ via product arcs in $C$. To be more specific, let $h_{0}^{1}$ be a 1-handle which meets $F \times\{0\}$. Let $h_{0}^{0}, h_{1}^{0}, h_{0}^{2}, h_{1}^{2}$, and $h_{2}^{2}$ be the 0 - and 2-handles meeting $h_{0}^{1}$ and $h_{1}^{1}$, $h_{2}^{1}$ and $h_{3}^{1}$ the 1-handles meeting $h_{0}^{0}$. We may assume that $\left(h_{0}^{2} \cup h_{3}^{1}\right) \cap \partial N=\varnothing$. For $i=0,1$, let $E_{i}$ be a disk in $\operatorname{Int}\left(R_{i} \cap \partial N\right), g_{i}=E_{i} \times[0,1], G_{i}=\left(\partial E_{i}\right) \times[0,1]$, and $\mu_{i}=x_{i} \times[0,1]$ for $x_{i} \in \operatorname{Int} E_{i}$, where the product structure refers to $C$.

We shall need the following additional properties of our special handle decomposition.

6.2 LEMMA. With the notation as above:

(7) for any 0-handle $h_{i}^{0}, H^{\prime}-h_{i}^{0}-h_{0}^{1}$ is connected,

(8) $H^{\prime \prime} \cup h_{0}^{1}$ is a cube with handles which meets $\partial N$ in a disjoint set of disks,

(9) for any 0-handle $h_{i}^{0}, H^{\prime \prime} \cup h_{i}^{0} \cup h_{0}^{1}$ is a cube with handles which meets $\partial N$ in a disjoint set of disks, and

(10) $H^{\prime \prime} \cup h_{0}^{0} \cup h_{0}^{1} \cup h_{1}^{1} \cup h_{2}^{1} \cup h_{3}^{1}$ is a cube with handles which meets $\partial N$ in a disjoint set of disks.

Proof. (7) By (5) $H^{\prime}-h_{i}^{0}$ is connected. If $i=0$ the result is clear. If $i \neq 0$, then there is a 2-handle $h_{k}^{2}$ which meets $h_{0}^{1}$ but does not meet $h_{i}^{0}$. Since $h_{k}^{2} \cap\left(H^{\prime}-h_{i}^{0}-\right.$ $h_{0}^{1}$ ) is connected the result follows. 
(8) Since $H^{\prime \prime} \cap h_{0}^{1}$ is a 2-cell, $H^{\prime \prime} \cup h_{0}^{1}$ is a cube with handles. $H^{\prime \prime} \cap \partial N$ consists of disjoint disks, two of which are $h_{1}^{2} \cap \partial N$ and $h_{2}^{2} \cap \partial N$. $\left(H^{\prime \prime} \cup h_{0}^{1}\right) \cap \partial N$ is obtained from $H^{\prime \prime} \cap \partial N$ by joining these two disks by the disk $h_{0}^{1} \cap \partial N$ which meets each of them in a single arc and therefore consists of disks.

(9) By (6) $H^{\prime \prime} \cup h_{i}^{0}$ is a cube with handles meeting $\partial N$ in a disjoint set of disks. If $h_{i}^{0} \cap \partial N=\varnothing$, then the proof is the same as that of (8). Suppose $h_{i}^{0} \cap \partial N \neq \varnothing$. If $i=0$ or 1 , the result is clear. If $i \neq 0$ or 1 , then let $h_{k}^{2}, h_{l}^{2}$, and $h_{m}^{2}$ be the 2-handles which meet both $h_{i}^{0}$ and $\partial N$. $\left(H^{\prime \prime} \cup h_{i}^{0}\right) \cap \partial N$ is formed from $H^{\prime \prime} \cap \partial N$ by joining $h_{k}^{2} \cap \partial N, h_{l}^{2} \cap \partial N$, and $h_{m}^{2} \cap \partial N$ along the disk $h_{i}^{0} \cap \partial N$, which meets each of them in a single arc, to form a disk $D$. If $D$ does not meet $h_{0}^{1}$, then the proof is as in (8). At most one of $h_{k}^{2}, h_{l}^{2}$, and $h_{m}^{2}$ meets $h_{0}^{1}$. Thus $\left(H^{\prime \prime} \cup h_{i}^{0} \cup h_{1}^{0}\right) \cap \partial N$ is obtained from $\left(H^{\prime \prime} \cup h_{i}^{0}\right) \cap \partial N$ by joining some $h_{n}^{2} \cap \partial N$ to either $D$ or $h_{p}^{2} \cap \partial N$ along the disk $h_{0}^{1} \cap \partial N$ which meets each in exactly one arc. Thus the result follows.

(10) Let $X=h_{0}^{0} \cup h_{0}^{1} \cup h_{1}^{1} \cup h_{2}^{1} \cup h_{3}^{1} . X$ is a 3-cell such that $X \cap H^{\prime \prime}$ is an annulus with a centerline the same as a centerline $\sigma$ of $S_{3}$. We may therefore regard $X$ as a 2-handle attached to $H^{\prime \prime}$ along $\sigma$. The disk $C_{0}^{2}$ in $H^{\prime \prime}$ meets $\sigma$ transversely in a single point. From this it is easy to see that $H^{\prime \prime} \cup X$ is a cube with handles having genus one less than that of $H^{\prime \prime}$. Clearly $\left(H^{\prime \prime} \cup X\right) \cap \partial N$ is homeomorphic to $\left(H^{\prime \prime} \cup h_{0}^{0}\right) \cap \partial N$ and so by (6) is a disjoint set of disks. This completes the proof.

Now let $\left.\left.\tilde{F}=\left(F-E_{0}-E_{1}\right) \times\{1\}, \tilde{C}=\overline{(} C-g_{0}-g_{1}\right), \tilde{R}_{i}=\overline{(} R_{i}-E_{i}\right) \cup\left(R_{i} \cap\right.$ $h_{0}^{1}$ ) for $i=0,1$, and $R_{i}$ for $i \geqslant 2$. Let $\tilde{R}=\cup \tilde{R}_{i}$. Let $\tilde{Z}=\tilde{C} \cup H^{\prime \prime} \cup h_{0}^{1}$ and $\tilde{S}=\cup S_{j}-S_{0}$.

\subsection{LEMMA. $\tilde{R}$ is incompressible in $\tilde{Z}$.}

Proof. Suppose $D$ is a compressing disk for $\tilde{R}_{i} . \partial D=\partial D^{\prime}$ for a disk $D^{\prime}$ in $\partial h_{i}^{0}$. Let $S=D \cup D^{\prime}$.

Case 1. $i=0$ or 1 , say $i=0$. $\tilde{Z} \cup h_{0}^{0}=\tilde{C} \cup H^{\prime \prime} \cup H^{\prime \prime} \cup h_{0}^{0} \cup h_{0}^{1}$. By (9) $H^{\prime \prime} \cup$ $h_{0}^{0} \cup h_{0}^{1}$ is a cube with handles meeting $\tilde{C}$ in a disjoint set of disks in $\tilde{F} \times\{0\}$. By Lemma $2.2 \tilde{Z} \cup h_{0}^{0}$ is irreducible. So $S$ bounds a 3-cell $B$ in $\tilde{Z} \cup h_{0}^{0}$. Since by (7) $H^{\prime}-h_{0}^{0}-h_{0}^{1}$ is connected, it must lie in $B$ or $(M-B)$. Thus $h_{0}^{0} \cap\left(H^{\prime}-h_{0}^{0}-h_{0}^{1}\right)$ lies in $D^{\prime}$ or $\tilde{R}_{0}-D^{\prime}$. So either $D^{\prime} \subseteq \tilde{R}_{0}$, and we are done, or $\partial D$ is parallel in $\tilde{R}_{0}$ to $\partial E_{0}$. If the latter is true, then $g_{0} \subseteq(M-B)$ and so $H^{\prime}-h_{0}^{0}-h_{0}^{1} \subseteq B$. But this is impossible since $S \cap g_{1}=\varnothing$ and $g_{1}$ joins $H^{\prime}-h_{0}^{0}$ to $\partial M$.

Case 2. $i \neq 0$ or 1. By (9) $H^{\prime \prime} \cup h_{i}^{0} \cup h_{0}^{1}$ is a cube with handles meeting $\tilde{C}$ in a disjoint set of disks in $\tilde{F} \times\{0\}$. It follows that $\tilde{Z} \cup h_{i}^{0}=\tilde{C} \cup H^{\prime \prime} \cup h_{i}^{0} \cup h_{0}^{1}$ is irreducible. So $S$ bounds a 3-cell $B$ in $\tilde{Z} \cup h_{i}^{0}$. By (7) $H^{\prime \prime}-h_{i}^{0}-h_{0}^{1}$ is connected and so must lie in $B$ or $(M-B)$. In either case $h_{i}^{0} \cap\left(H^{\prime}-h_{0}^{1}-h_{i}^{0}\right)$ lies in $D^{\prime}$ or $\overline{\partial h_{i}^{0}-D^{\prime}}$ and so $\partial D=\partial D^{\prime \prime}$ for some disk $D^{\prime \prime}$ in $R_{i}$. This completes the proof.

6.4 LEMMA. $(\bar{\partial} \tilde{Z}-\tilde{R})$ is incompressible in $\tilde{Z}$, which is irreducible.

Proof. The incompressibility of $\tilde{S}$ follows from that of $\tilde{R}$. By (8) $H^{\prime \prime} \cup h_{0}^{1}$ is a cube with handles meeting $\tilde{C}$ in a disjoint set of disks in $\tilde{F} \times\{0\}$. The result now follows from Lemma 2.2 .

6.5 Lemma. ( $\tilde{Z}, \tilde{R})$ has Property A. 
Proof. Let $D$ be a disk in $\tilde{Z}$ with $\partial D \cap \tilde{R}$ a single $\operatorname{arc} \alpha$. Let $\beta=\overline{(\partial D}-\alpha)$.

Suppose $\alpha \cap\left(g_{0} \cup g_{1}\right)=\varnothing$. Then $\beta$ is a boundary-parallel arc in $\tilde{S}$. So $D$ can be isotoped so that $D \subseteq \tilde{R}$ and is therefore boundary-parallel by the irreducibility of $\tilde{Z}$ and incompressibility of $\tilde{R}$.

Suppose $\alpha \cap\left(g_{0} \cup g_{1}\right) \neq \varnothing$. We may assume that $\alpha \subseteq R_{0}, \partial \alpha \subseteq \partial E_{0}$, and $\beta=$ $\beta_{0} \cup \tilde{\beta} \cup \beta_{1}$, where $\beta_{n}=y_{n} \times[0,1]$ for $y_{n} \in \partial E_{0}, n=0,1$, and $\tilde{\beta} \subseteq \tilde{F} \times\{1\}$. If $\alpha$ is boundary-parallel in $\tilde{R}_{0}$ or $\beta$ is boundary-parallel in $\tilde{F}$, then $D$ can be isotoped so that $\partial D$ is in, respectively, $\tilde{F}$ or $\tilde{R}_{0}$. As above, this implies that $D$ is boundaryparallel. So we may assume that neither of these is the case.

By (10) $H^{\prime \prime} \cup h_{0}^{0} \cup h_{0}^{1} \cup h_{1}^{1} \cup h_{2}^{1} \cup h_{3}^{1}$ is a cube with handles which meets $\left(\tilde{F} \cup E_{0}\right) \times[0,1]$ in a disjoint collection of disks in $\left(\tilde{F} \cup E_{0}\right) \times\{0\}$. Therefore $\left(\tilde{F} \cup E_{0}\right) \times\{1\}$ is incompressible in

$$
\begin{aligned}
\tilde{Z} \cup g_{0} \cup h_{0}^{0} \cup h_{1}^{1} \cup h_{2}^{1} \cup h_{3}^{1} \\
=\left(\left(\tilde{F} \cup E_{0}\right) \times[0,1]\right) \cup\left(H^{\prime \prime} \cup h_{0}^{0} \cup h_{0}^{1} \cup h_{1}^{1} \cup h_{2}^{1} \cup h_{3}^{1}\right),
\end{aligned}
$$

which is irreducible. The latter 3-manifold is homeomorphic to $\tilde{Z} \cup h_{1}^{1} \cup h_{2}^{1} \cup h_{3}^{1}$ by a homeomorphism which takes $\left(\tilde{F} \cup E_{0}\right) \times\{1\}$ to $\tilde{F} \cup G_{0} \cup\left(\partial h_{0}^{0}-E_{0}\right)$. Therefore $\tilde{F} \cup G_{0} \cup\left(\partial h_{0}^{0}-E_{0}\right)$ is incompressible in the irreducible 3-manifold $\tilde{Z} \cup h_{1}^{1} \cup h_{2}^{1}$ $\cup h_{3}^{1}$.

Thus $\partial D=\partial D^{\prime}$, where $D^{\prime}$ is a disk in $\left.\tilde{F} \cup G_{0} \cup \overline{(} \partial h_{0}^{0}-E_{0}\right) .\left(\partial E_{0} \times\{1\}\right) \cap D^{\prime}$ separates $D^{\prime}$ into two subdisks, one of which lies in $\tilde{F}$ and is bounded by the union of this arc and $\tilde{\beta}$. Therefore $\tilde{\beta}$ is boundary-parallel in $\tilde{F}$, a contradiction. This completes the proof.

The proof of the following lemma is straightforward and therefore is left to the reader. A spanning arc in an annulus $A$ is a properly embedded, nonboundary parallel arc.

6.6 Lemma. Let $M$ be a compact, orientable, irreducible 3-manifold and $F$ an incompressible surface in $\partial M$ such that $(\partial M-F)$ is incompressible. Let $D_{1} \cdots D_{n}$ be a disjoint collection of disks in $M$ whose boundaries are in general position with respect to $\partial F$. Let $D=D_{1} \cup \cdots \cup D_{n}$. Let $A$ be an incompressible annulus in $M$ which is in general position with respect to $D$ and such that $\partial A \cap \partial F=\varnothing$. If, among all such annuli isotopic to $A$ rel $\partial F, A \cap D$ has a minimal number of components, then $A \cap D$ consists of at most spanning arcs of $A$.

Now let $\xi_{0}, \xi_{1}$, and $\xi_{2}$ be disjoint, parallel arcs in $\operatorname{Int}\left(\left(\tilde{R}_{0} \cup h_{0}^{1} \cup \tilde{R}_{1}\right) \cap \partial N\right)$ which join $\partial E_{0}$ and $\partial E_{1}$ and have connected intersection with $h_{0}^{1}$. Number so that the $\xi_{i} \cap h_{0}^{1}$ can be joined to the $C_{i}^{2} \cap h_{0}^{1}$ by disjoint disks $D_{i}$ in $h_{0}^{1}, i=0,1,2$. Let $\tilde{C}_{i}^{2}=C_{i}^{2} \cup D_{i} \cup\left(\xi_{i} \times[0,1]\right)$. Let $\tilde{h}_{i}^{2}$ be a regular neighborhood of $\tilde{C}_{i}^{2}$ in $\tilde{Z}$, chosen so that a product structure $\tilde{h}_{i}^{2}=\tilde{C}_{i}^{2} \times[-1,+1]$ is compatible with the product structure $h_{i}^{2}=C_{i}^{2} \times[-1,+1]$. The $\tilde{h}_{i}^{2}$ are assumed disjoint. For $i \geqslant 3$, let $\tilde{h}_{i}^{2}=h_{i}^{2}$ and $\tilde{C}_{i}^{2}=C_{i}^{2}$.

6.7 Lemma. ( $\tilde{Z}, \tilde{R})$ has Property $\mathrm{B}^{\prime}$. 
Proof. Let $G$ be an incompressible surface in $\dot{Z}$ such that either $\partial G=\varnothing$ or $\partial G \subseteq(\partial M-F) \cup\left(\tilde{F} \cup G_{0} \cup G_{1}\right)$. We may assume that $\partial G \cap\left(G_{0} \cup G_{1}\right)=\varnothing$ and that among all surfaces in $\tilde{Z}$ isotopic to $G$ rel $\partial \tilde{F}, G \cap\left(\left(H^{\prime \prime} \cup h_{0}^{1}\right) \cap \tilde{C}\right)$ has a minimal number of components. Since $\left(H^{\prime \prime} \cup h_{0}^{1}\right) \cap \tilde{C}$ is a disjoint set of disks, $G$ lies in $\tilde{C}$, and so, by Corollary 3.2 of [14], is parallel to a surface in $\tilde{C} \cap \partial M$. In particular every incompressible torus and every incompressible annulus with boundary in $\overline{(\partial \tilde{Z}}-\tilde{R}-\tilde{S})$ is boundary-parallel.

Suppose $A$ is an incompressible annulus in $\tilde{Z}$ with boundary components $\alpha$ and $\beta$ such that $\partial A$ is not contained in $(\partial \tilde{Z}-\tilde{R}-\tilde{S})$. We may assume that $A \cap \tilde{S}=\varnothing$, that $A$ and $\cup \tilde{C}_{i}^{2}$ are in general position, and that $A \cap\left(\cup \tilde{C}_{i}^{2}\right)$ has a minimal number of components.

Case 1. $\alpha \subseteq \tilde{R}_{i}$ and $\beta \subseteq \tilde{F}$. Suppose $i \neq 0$ or 1 . Then at least two 2-handles, $h_{k}^{2}$ and $h_{l}^{2}$, meet $A$ and a common 1-handle $h_{j}^{1}$. At most one of these, say $h_{k}^{2}$, meets $h_{0}^{1}$. $A \cap \tilde{C}_{k}^{2}$ contains a spanning arc $\gamma$ running from $R_{i}$ to $\tilde{F} . A \cap C_{l}^{2}$ also contains a spanning arc $\delta$ running from $R_{i}$ to $\tilde{F}$, but this is impossible because $h_{l}^{2}$ does not meet $h_{0}^{1}$.

Suppose $i=0$ or 1 , say $i=0$. If $\alpha$ is parallel to $\partial E_{0}$ in $\tilde{R}_{0}$, then $A$ can be isotoped so that $\partial A \subseteq \tilde{F}$, and therefore $A$ is boundary-parallel. If $\alpha$ is parallel in $\tilde{R}_{0}$ to $\partial\left(h_{j}^{1} \cap h_{i}^{0}\right)$ for some $j \neq 0$, then it meets the three 2-handles incident with $h_{j}^{1}$. At most one of these meets $h_{0}^{1}$; let $h_{k}^{2}$ be one which does not. Then $A \cap C_{k}^{2}$ contains a spanning arc $\gamma$ joining $\tilde{R}_{0}$ with $\tilde{F}$, but this is impossible since $h_{k}^{2}$ does not meet $h_{0}^{1}$. If $\alpha$ is not boundary-parallel in $\tilde{R}_{0}$, then it meets at least two 2 -handles which meet a common 1-handle other than $h_{0}^{1}$. One of these, say $h_{k}^{2}$, does not meet $h_{0}^{1}$. But $A \cap C_{k}^{2}$ contains a spanning $\operatorname{arc} \gamma$ joining $\tilde{R}_{0}$ to $\tilde{F}$. Again, this is impossible because $h_{k}^{2}$ does not meet $h_{0}^{1}$.

Case 2. $\alpha \subseteq \tilde{R}_{i}$ and $\beta \subseteq \tilde{R}_{m}$. If $i=m$, then $A$ is $\tilde{R}_{i}$-compressible and thus $\tilde{R}_{i}$-parallel, because $A$ intersects some $C_{k}^{2}$ in a spanning, $R_{i}$-parallel arc. If either $\alpha$ or $\beta$ is parallel in $\tilde{R}$ to some $\partial E_{i}$, then $A$ can be isotoped so that one component of $\partial A$ is in $\tilde{R}$ and the other in $\tilde{F}$. Thus $A$ is boundary-parallel by the previous case. We therefore assume that neither of these conditions occurs.

Subcase (a). $\alpha$ is boundary-parallel in $\tilde{R}_{i}$. Then $\alpha$ is parallel to $\partial\left(h_{i}^{0} \cap h_{j}^{1}\right)$ for some $j \neq 0$. $\alpha$ meets all three 2-handles incident with $h_{j}^{1}$. At most one of these meets $h_{0}^{1}$. Let $h_{k}^{2}$ and $h_{l}^{2}$ be the others. $A \cap C_{k}^{2}$ contains a spanning $\operatorname{arc} \gamma$ which joins $\tilde{R}_{i}$ to $\tilde{R}_{m}$. $A \cap C_{l}^{2}$ contains a similar arc $\delta$. It follows that $h_{j}^{1}$ joins $h_{i}^{0}$ and $h_{m}^{0}$. If $\beta$ is not parallel in $\tilde{R}_{m}$ to $\partial\left(h_{m}^{0} \cap h_{j}^{1}\right)$, then $\beta$ meets some $\tilde{h}_{n}^{2}$ which does not meet $h_{j}^{1} . A \cap \tilde{C}_{n}^{2}$ contains a spanning arc $\varepsilon$ joining $\tilde{R}_{m}$ to $\tilde{R}_{i}$. But since $\tilde{h}_{n}^{2}$ does not meet $h_{j}^{1}$, neither does $h_{n}^{2}$. But this is impossible since it meets both $h_{i}^{0}$ and $h_{m}^{0}$. Therefore $\beta$ is parallel in $\tilde{R}_{m}$ to $\partial\left(h_{m}^{0} \cap h_{j}^{1}\right)$. Thus $A$ is $S_{j}^{\prime}$-compressible, where $S_{j}^{\prime}$ is the union of $S_{j}$ with the boundary-parallelisms of $\alpha$ and $\beta$ in $\tilde{R}_{i}$ and $\tilde{R}_{m}$, respectively. Thus $A$ is boundaryparallel.

Subcase (b). $\alpha$ is not boundary-parallel in $\tilde{R}_{i}$. Then $\alpha$ meets at least four distinct $\tilde{h}_{n}^{2}$. Let $\gamma_{1}, \gamma_{2}, \gamma_{3}, \gamma_{4}$ be arcs in the intersection of $A$ with the corresponding $\tilde{C}_{n}^{2}$. Each $\gamma_{q}$ joins $\tilde{R}_{i}$ to $\tilde{R}_{m}$, so each of the $\tilde{h}_{n}^{2}$ meets both $h_{i}^{0}$ and $h_{m}^{0}$. Therefore each of the $h_{n}^{2}$ meets both $h_{i}^{0}$ and $h_{m}^{0}$. Thus $h_{i}^{0}$ and $h_{m}^{0}$ are joined by a l-handle $h_{j}^{1}$ which meets each 
of the four distinct $h_{n}^{2}$. This contradicts the fact that each 1-handle meets exactly three distinct 2-handles.

Proof of Proposition 6.1. Let $\left(\lambda_{1, i}, \lambda_{2, i}\right)$ be a copy of the true lover's tangle in a 3-cell $B_{i}$. Let $Q_{i}$ be the associated tangle space. For $i \geqslant 2$, identify each 0 -handle $h_{i}^{0}$ with $B_{i}$ in such a way that $\partial\left(\lambda_{1, i} \cup \lambda_{2, i}\right)$ is identified with the intersection of $h_{i}^{0}$ and the cores $C_{j}^{1}$ of the four 1-handles meeting $h_{i}^{0}$. For $i=0,1$, identify $h_{i}^{0}$ with $B_{i}$ in such a way that the three points of $\partial\left(\lambda_{1, i} \cup \lambda_{2, i}\right)$ are identified with the intersections of $h_{i}^{0}$ with the cores $C_{j}^{1}$ of the three 1-handles other than $h_{0}^{1}$ which meet $h_{i}^{0}$. Identify the remaining point of $\partial\left(\lambda_{1, i} \cup \lambda_{2, i}\right)$ with $h_{i}^{0} \cap \mu_{i}$. Perform all these identifications in such a manner that

$$
\tilde{J}=\bigcup_{i}\left(\lambda_{1, i} \cup \lambda_{2, i}\right) \underset{j \neq 0}{\cup} C_{j}^{1} \cup \mu_{0} \cup \mu_{1}
$$

is a single arc.

Let $Q=\cup_{i} Q_{i}$. Then the exterior $\tilde{X}$ of $\tilde{J}$ is $Q \cup \tilde{Z}$. Note that $Q \cap \tilde{Z}=\tilde{R}$. By Proposition 4.1 $Q$ is a simple Haken manifold. Since $\tilde{R}$ and $(\partial Q-\tilde{R})$ are incompressible in $Q,(Q, \tilde{R})$ has Property $\mathrm{C}^{\prime}$. By Lemma $6.7(\tilde{Z}, \tilde{R})$ has Property $\mathrm{B}^{\prime}$. Thus by Lemma $2.5 \tilde{X}$ is a simple Haken manifold.

6.8. Question. Let $M$ be as in Proposition 6.1. If $\partial M$ contains two different components $F_{0}$ and $F_{1}$, does there exist a properly embedded arc $\tilde{J}$ in $M$ joining $F_{0}$ and $F_{1}$ whose exterior is a simple Haken manifold?

\section{Relative homology cobordisms and link concordances.}

7.1 TheOREM. Let $M_{0}^{3}$ be a compact, connected, oriented 3-manifold such that $\partial M^{3} \neq \varnothing$ and contains no 2-spheres. Then $M_{0}^{3}$ is relative homology cobordant to a hyperbolic 3-manifold $M_{1}^{3}$.

7.2 THEOREM. Let $M^{3}$ be a compact, connected, oriented 3-manifold such that $\partial M^{3}$ contains no 2-spheres. Then every link $L_{0}$ in $M^{3}$ is concordant to a link $L_{1}$ with hyperbolic exterior.

7.3 Corollary. Every knot $K_{0}$ in $S^{3}$ is concordant to a knot $K_{1}$ with hyperbolic exterior.

7.4 COROllaRy (KIRBY AND LiCKORISH [7], LivingSTON [8]). Every knot $K_{0}$ in $S^{3}$ is concordant to a prime knot $K_{1}$.

We shall give a unified proof of these theorems by means of

7.5 Proposition. Let $X^{3}$ be a compact, connected, oriented 3-manifold such that $\partial X^{3}$ contains no 2-spheres. Then every wedge of circles $G_{0}$ in Int $X^{3}$ is concordant to a wedge of circles $G_{1}$ in Int $X^{3}$ whose exterior is hyperbolic.

Proof. Let $Y^{3}$ be the exterior of $G_{0}$ in $X^{3}$. Let $F^{2}$ be the component of $\partial Y^{3}$ which bounds $\left.H^{3}=\overline{(} X^{3}-Y^{3}\right)$. Let $\mu_{1}^{\prime}$ and $\mu_{2}^{\prime}$ be disjoint arcs in the same circle of $G_{0}$, chosen disjoint from the wedge point. Let $E_{1}^{3}$ and $E_{2}^{3}$ be disjoint regular neighborhoods of $\mu_{1}^{\prime}$ and $\mu_{2}^{\prime}$ which meet $H^{3}$ in annuli $A_{1}^{2}$ and $A_{2}^{2}$, respectively. By Proposition 6.1 there is a properly embedded arc $\tilde{J}$ in $Y^{3}$ whose exterior $W^{3}$ is a simple Haken 
manifold and such that $\partial \tilde{J} \subseteq F^{2}$. Let $\left.Z^{3}=\overline{(} Y^{3}-W^{3}\right)$. We may assume that $Z^{3} \cap H^{3}$ consists of one disk in each of $A_{1}^{2}$ and $A_{2}^{2}$.

Let $\left\{\mu_{1}, \mu_{2}\right\}$ be the composition of the true lover's tangle $\left\{\lambda_{1}, \lambda_{2}\right\}$ with its reflection in a plane, as in $\S 5$. Identify $B^{3}$ with $E_{1}^{3} \cup Z^{3} \cup E_{2}^{3}$ in such a way that $\partial \mu_{1}=\partial \mu_{1}^{\prime}$ and $\partial \mu_{2}=\partial \mu_{2}^{\prime}$. Let $G_{1}=\left(G_{0}-\mu_{1}^{\prime}-\mu_{2}^{\prime}\right) \cup \mu_{1} \cup \mu_{2} .\left\{\mu_{1}, \mu_{2}\right\}$ is concordant in $B^{3}$ to the trivial tangle $\left\{\mu_{1}^{\prime}, \mu_{2}^{\prime}\right\}$. Therefore $G_{0}$ and $G_{1}$ are concordant in $X^{3}$.

Let $Q^{3}$ be the tangle space of $\left\{\mu_{1}, \mu_{2}\right\} . Q^{3} \cap W^{3}$ is a 2 -sphere with four holes which is an incompressible subsurface of both $\partial Q^{3}$ and $\partial W^{3} \cdot Q^{3}$ is a simple Haken manifold by Proposition 4.1. $W^{3}$ is a simple Haken manifold by Proposition 6.1. Thus both $\left(Q^{3}, Q^{3} \cap W^{3}\right)$ and $\left(W^{3}, Q^{3} \cap W^{3}\right)$ have Property $\mathrm{C}^{\prime}$. Therefore by Lemma 2.5, the exterior $Q^{3} \cup W^{3}$ of $G_{1}$ is a simple Haken manifold and thus is hyperbolic.

Proof of 7.1. Let $F^{2}$ be a component of $\partial M_{0}^{3}$. Let $H^{3}$ be a cube with handles such that $\partial H^{3}$ is homeomorhic to $F^{2}$. Construct $X^{3}$ by attaching $H^{3}$ to $M_{0}^{3}$ via this homeomorphism. Let $G_{0}$ be a wedge of circles of which $H^{3}$ is a regular neighborhood in $X^{3}$. By Proposition $7.5 G_{0}$ is concordant to $G_{1}$ such that the exterior $M_{1}^{3}$ of $G_{1}$ in $X^{3}$ is hyperbolic. By Lemma 2.7 the exterior $\left(P^{4} ; M_{0}^{3}, M_{1}^{3}\right)$ of this concordance is a relative homology cobordism.

Proof OF 7.2. Let $K_{1}, \ldots, K_{n}$ be the components of $L_{0}$, with disjoint regular neighborhoods $U_{1}, \ldots, U_{n}$ in $M^{3}$. Let $\left.X^{3}=\overline{(} M^{3}-\left(U_{2} \cup \cdots \cup U_{n}\right)\right)$ and $G_{0}=K_{1}$. By Proposition $7.5 G_{0}$ is concordant in $X^{3}$ to $G_{1}$ such that the exterior of $G_{1}$ in $X^{3}$ is hyperbolic. By taking product concordances $K_{i} \times[0,1]$ in $U_{i} \times[0,1]$ for $i \geqslant 2$, we see that $L_{0}=\left\{G_{0}, K_{2}, \ldots, K_{n}\right\}$ is concordant in $M^{3}$ to $L_{1}=\left\{G_{1}, K_{2}, \ldots, K_{n}\right\}$. The exterior of $L_{1}$ in $M^{3}$ is the same as that of $G_{1}$ in $X^{3}$ and so is hyperbolic.

\section{REFERENCES}

1. R. H. Bing, Necessary and sufficient conditions that a 3-manifold be $S^{3}$, Ann. of Math. (2) 68 (1958), 17-37.

2. D. Galewski and R. Stern, Classification of simplicial triangulations of topological manifolds, Ann. of Math. (2) 111 (1980), 1-34.

3. G. González-Acuña, 3-dimensional open books, Lectures Univ. of Iowa Topology Seminar, 1974/75.

4. J. Hempel, 3-manifolds, Ann. of Math. Studies, no. 86, Princeton Univ. Press, Princeton, N.J., 1976.

5. W. Jaco, Lecture on three-manifold topology, CBMS Regional Conf. Ser. in Math., no. 43, Amer. Math. Soc., Providence, R.I., 1980.

6. W. Jaco and P. Shalen, Seifert fibered spaces in 3-manifolds, Mem. Amer. Math. Soc., No. 220 (1979).

7. R. C. Kirby and W. B. R. Lickorish, Prime knots and concordances, Math. Proc. Cambridge Philos. Soc. 86 (1979), 437-441.

8. C. Livingston, Homology cobordisms of 3-manifolds, Pacific J. Math. 94 (1981), 193-206.

9. T. Matumoto, Triangulations of manifolds, Proc. Sympos. Pure Math., vol. 32, Amer. Math. Soc., Providence, R.I., 1977, pp. 3-6.

10. R. Myers, Open book decompositions of 3-manifolds, Proc. Amer. Math. Soc. 72 (1978), 397-402.

11. Simple knots in compact, orientable 3-manifolds, Trans. Amer. Math. Soc. 273 (1982), 75-92.

12. D. Rolfsen, Knots and links, Publish or Perish, Berkeley, Calif., 1976.

13. W. H. Row, An algebraic characterization of connected sum factors of closed 3-manifolds, Trans. Amer. Math. Soc. 250 (1979), 347-356.

14. F. Waldhausen, On irreducible 3-manifolds which are sufficiently large, Ann. of Math. 87 (1968), $56-88$.

Department of Mathematics, Oklahoma State University, Stillwater, Oklahoma 74078 